\title{
The Future of Infectious Diseases Education
}

\author{
Jorge Cervantes ${ }^{1}$
}

Published online: 13 July 2020

(C) International Association of Medical Science Educators 2020

\begin{abstract}
The infectious diseases (ID) specialty continues to be in crisis due to a lack of interest by residents in the recruitment pool. It is possible that enhancing and innovating how microbiology is taught in medical school could increase the interest in ID. We need to improve teaching of preclinical medical microbiology and immunology by incorporating advancements in education and learning technologies to reignite the interest in the field. The shortage of ID specialists has important implications in our fight against ID threats such as the current COVID-19 global pandemic. In order to address this problem, we need to perform extensive research to identify the issues that medical learners and trainees are facing as they progress through their medical education towards the ID specialty.
\end{abstract}

Keywords Infectious disease $\cdot$ Microbiology $\cdot$ Immunology $\cdot$ Experiential learning $\cdot$ Inter-professional

\section{The Crisis of Infectious Diseases Specialists}

For the past several years, there has been a steady decline in the number of internal medicine residents applying for fellowship positions in infectious diseases (ID) [1]. Of 406 ID fellowships offered, 86 were not filled during the 2020 National Resident Matching Program [2]. A majority of residents remain uninterested, with most changing their mind later after initially considering ID, citing salary, lack of procedures, and training length as primary deterrents to choosing ID [3, 4]. Although ID appears to be preferred over other specialties like geriatrics in countries like France [5], it is unknown if the lack of interest in ID is a phenomenon occurring worldwide or restricted to the USA, as this seems to be an understudied issue.

It is possible that improving and innovating how microbiology is taught in medical school could in turn improve the interest in ID. It was previously reported that a greater percentage of graduating internal medicine residents that applied to ID thought that the quality of their medical microbiology curriculum was very good, and that the quality of their preclinical training was very influential in the level of interest in

Jorge Cervantes

jorge.cervantes@ttuhsc.edu

1 Department of Medical Education, Paul L. Foster School of Medicine, Texas Tech University Health Sciences Center at El Paso, El Paso, TX, USA
ID [6]. The majority of residents, especially those who selected ID as a career, stated that they became interested in their chosen field before or during medical school [7].

In a world threatened by longstanding, emerging, and reemerging IDs, a new generation of well-trained ID specialists is needed to tackle the challenging work of providing correct diagnoses, choosing antibiotics wisely, and understanding when to stop them $[1,8]$. Immunology education has historically been tightly linked to ID, but with advancements in this field (e.g., cancer immunotherapies, inflammatory syndromes, and autoimmune diseases) its importance in several clinical specialties goes beyond protective immunity [9]. Therefore, educating the next generation of physicians in the field of ID would have an enormous impact on the application of new scientific knowledge to human diseases in various fields [10].

Reforms in microbiology curricula at medical schools [11] should be developed, providing first-, second-, or third-year medical students with access to an ID experience designed to spark intellectual curiosity and inspire the pursuit of a career in the field of ID. Exploring ID concepts, inside or outside of their primary institution [12], via the use of technology could have a positive effect in the much needed supply of new ID specialists.

\section{Teaching Modalities}

Methods of teaching basic science in medical schools are rapidly changing [13]. Regarding the structure of medical school 
ID learning sessions, it has been previously reported that those who applied to ID belonged to a medical school with a curriculum that moved away from traditional lectures and had higher proportion of case-based and laboratory-based training, compared with those that only considered ID or were uninterested [6]. Furthermore, this same study also showed that mentorship, scholarship, and/or conference attendance positively influenced entry into a chosen field.

The Infectious Diseases Society of America (IDSA) has provided guidelines for improving the teaching of preclinical medical microbiology and ID [14]. They also have prepared a detailed report on factors that are likely to influence the future supply and demand for ID physicians and related projections [11]. These recommendations try to distance ID education from utilization of PowerPoint lectures and multiple choice tests, which encourage short-term memory, without promoting understanding and long-term memory. Strategies such as incorporating real-time teaching, peer instruction of large group sessions, and small-group discussions promise to enhance learning and reignite the interest in the field of ID [14]. In fact, peer teaching for medical microbiology lectures has shown an improvement of students' level of understanding, satisfaction, and attitude toward learning [15].

Identifying learning platforms that could facilitate effective knowledge transfer with improved quality and efficiency is one of the aims proposed by the Global Independent Commission for Education of Health Professionals [16]. This international commission's proposed framework and strategy is not specific to ID, but rather aims for education improvement in medicine as a whole [16]. Instructional reforms have helped the continuous development of medical education, moving from a science-based curriculum, toward problem-based learning and a system-based competency [16].

\section{How Technological Innovation Impacts Microbiology Teaching and Learning Experiences}

Technological advances are making processes and products more efficient, making the way we acquire information much faster. Innovations in health have also exploded in the last decade with devices that connect to the Internet, allowing for easy dissemination of information. Within medical education, microbiology and immunology have greatly benefited from online learning, and the use of electronic media [17], has shown to improve medical students' microbiology examination performance and engagement [18]. Technology should be used to support the ongoing development of learners from undergraduate medical education through clinical practice. Detailed recommendations on the use of education technologies in health professions have been provided by the Josiah Macy Jr. Foundation [19]. These describe the use of technologies for face-to-face, online, and simulation-based instruction, as well as technologies for assessment, and integration with clinical practice [19].

Learners from this new generation, as well as educators, need to be prepared for a new health system that relies heavily on technology. The adoption of innovative pedagogies complemented by educational technologies that promote active engagement in the learning process [20] may be a challenging process. Increasing demands on faculty time, expanding class sizes and curricula, and a desire to increase accessibility while ensuring affordability threatens innovation in health education [21]. To these drawbacks, we should add e-learning's perceived usefulness, and in some cases ease of utilization (compared with widely used platforms such as Google) [10].

There are several ways to enhance medical education through the incorporation of state of the art technology in teaching, learning, and assessment [22, 23]. Since educational technologies include materials and devices used to solve practical problems related to training and learning, the utilization of novel and practical technological devices can also have an impact on the experiential learning of basic sciences such as microbiology and immunology. Low-cost devices can help medical students' interests in scientific advances, such as next-generation sequencing and its numerous applications in medicine [24]. Science platforms with a microbiological focus [22], along with teaching support from university research laboratories [25] and the application of bioengineering, can also improve microbiology learning [26]. Experiential learning of medical students undertaking a microbiology/ immunology research project has shown subjective attitude changes after the experiential learning (i.e., increased science motivation, confidence, and knowledge), and developing a new social niche [27]. When aligned with organizational goals, experiential learning can help achieve improvements in quality and safety at the clinical level while also improving the education and competency of trainees [28].

In an era of globalization, modern medical education needs to build opportunities for developing a global health workforce that understands the role of culture, scientific and technological literacy, and new effective approaches to health and disease. As health education becomes increasingly globalized, standardization of knowledge, skill, and professionalism competencies will become necessary. Teaching quality, with transmission of knowledge, skills, and attitudes with assessment of processes rather than content, will be the new paradigm to provide future physicians with the critical thinking required to adapt and thrive within this new environment.

The shortage of ID specialists has important implications in our fight against ID threats such as the current COVID-19 global pandemic [29]. In order to address this problem, we need to perform extensive research to identify the issues that 
medical learners and trainees are facing as they progress through their medical education toward the ID specialty.

Acknowledgments Special thanks for Michelle Castro (PLFSOM, TTUHSCEP) for her assistance in editing the manuscript.

Authors' Contributions JC prepared the manuscript.

\section{Compliance with Ethical Standards}

Conflict of Interest The authors declare that they have no conflict of interest.

\section{References}

1. Donskey C. Never let a crisis go to waste: recruiting the next generation of infectious diseases physicians. Pathog Immun. 2017;2(2): 270-3.

2. Program TNRM. NRMP Results and Data Specialties Matching Service, 2020 Appointment Year 2020.

3. Barsoumian AE, Hartzell JD, Bonura EM, Ressner RA, Whitman TJ, Yun HC. Factors influencing selection of infectious diseases training for military internal medicine residents. Clin Infect Dis. 2018;67(10):1582-7. https://doi.org/10.1093/cid/ciy322.

4. Chandrasekar P, Havlichek D, Johnson LB. Infectious diseases subspecialty: declining demand challenges and opportunities. Clin Infect Dis. 2014;59(11):1593-8. https://doi.org/10.1093/cid/ ciu656.

5. Bariety A. Medecine: les futurs internes preferent les maladies infectieuses a la geriatrie. Le Figaro etudiant. 2017.

6. Bonura EM, Lee ES, Ramsey K, Armstrong WS. Factors influencing internal medicine resident choice of infectious diseases or other specialties: a national cross-sectional study. Clin Infect Dis. 2016;63(2):155-63. https://doi.org/10.1093/cid/ciw263.

7. Bonura EM, Armstrong WS. Increasing subspecialization in the field of infectious diseases: evaluating challenges and strategies to move forward. J Infect Dis. 2017;216(suppl_5):S594-S9. https:// doi.org/10.1093/infdis/jix316.

8. Bloom DE, Cadarette D. Infectious disease threats in the twentyfirst century: strengthening the global response. Front Immunol. 2019;10:549. https://doi.org/10.3389/fimmu.2019.00549.

9. Bruns HA, Deaver J, Justement LB. Out of the curricular shadows: revolutionizing undergraduate immunology education. Front Immunol. 2019;10:2446. https://doi.org/10.3389/fimmu.2019. 02446.

10. Haidaris CG, Frelinger JG. Inoculating a new generation: immunology in medical education. Front Immunol. 2019;10:2548. https://doi.org/10.3389/fimmu.2019.02548.

11. IDSA TGWUHWIa. The Future Supply and Demand for Infectious Disease Physician 2017.

12. Charlier C, Johannessen I, Mackintosh CL, Wilks D, Cauda R, Wolf FI, et al. International infectious diseases teaching to undergraduate medical students: a successful European collaborative experience. Med Teach. 2017;39(9):981-6. https://doi.org/10.1080/ 0142159X.2017.1332364.

13. Berk SL. The infectious disease manpower crisis: finding the cure. Tex Med. 2017;113(2):60-2.

14. Southwick F, Katona P, Kauffman C, Monroe S, Pirofski LA, del Rio $\mathrm{C}$, et al. Commentary: IDSA guidelines for improving the teaching of preclinical medical microbiology and infectious diseases. Academic medicine : journal of the Association of American Medical Colleges. 2010;85(1):19-22. https://doi.org/10. 1097/ACM.0b013e3181c485c5.

15. Ngamskulrungro P, Kiratisin P, Dangprapai Y, Thaipisuttikul I, Leelaporn A, Luisirirojanakul S, et al. The efficacy of peer teaching for medical microbiology lectures. MedEdPublish. 2017;6(3):18.

16. Frenk J, Chen L, Bhutta ZA, Cohen J, Crisp N, Evans T, et al. Health professionals for a new century: transforming education to strengthen health systems in an interdependent world. Lancet. 2010;376(9756):1923-58. https://doi.org/10.1016/S01406736(10)61854-5.

17. Guarner J, Nino SM. Microbiology learning and education online. J Clin Microbiol. 2016;54(5):1203-8. https://doi.org/10.1128/JCM. 03176-15.

18. Stevens NT, Holmes K, Grainger RJ, Connolly R, Prior AR, Fitzpatrick F, et al. Can e-learning improve the performance of undergraduate medical students in clinical microbiology examinations? BMC Med Educ. 2019;19(1):408. https://doi.org/10.1186/ s12909-019-1843-0.

19. Foundation JMJ, editor. Enhancing health professions education through technology: building a continously learning health system 2015.

20. Huynh R. The role of E-learning in medical education. Academic medicine : journal of the Association of American Medical Colleges. 2017;92(4):430. https://doi.org/10.1097/ACM. 0000000000001596.

21. McDonald P. Are we prepared for the future of health care education? Online learning consortium. 2017.

22. Fahnert B. Edging into the future: education in microbiology and beyond. FEMS Microbiol Lett. 2016;363(7). https://doi.org/10. 1093/femsle/fnw048.

23. Jamil NA, Shaikh S, Munir S, Malek S, Khan A. Development of elearning in medical education: a student's perspective. Korean J Med Educ. 2019;31(4):371-3. https://doi.org/10.3946/kjme.2019. 147.

24. Cervantes J, Perry C, Wang M. Teaching next-generation sequencing to medical students with a portable sequencing device [published online ahead of print, 2020 Mar 3]. Perspect Med Educ. 2020. https://doi.org/10.1007/s40037-020-00568-2

25. Caine M, Zuchuat S, Weber A, Ducret V, Linder P, Perron K. BiOutils: an interface to connect university laboratories with microbiology classes in schools. FEMS Microbiol Lett. 2015;362(20). https://doi.org/10.1093/femsle/fnv171.

26. Kelwick R, Bowater L, Yeoman KH, Bowater RP. Promoting microbiology education through the iGEM synthetic biology competition. FEMS Microbiol Lett. 2015;362(16). https://doi.org/10. 1093/femsle/fnv129.

27. Cervantes J, Hong BY. The impact of experiential learning in hostpathogen research on medical students' interest and attitudes towards microbiology and immunology. Chicago: ASM 2020; 2020.

28. Gonzalo JD, Wolpaw D, Graaf D, Thompson BM. Educating patient-centered, systems-aware physicians: a qualitative analysis of medical student perceptions of value-added clinical systems learning roles. BMC Med Educ. 2018;18(1):248. https://doi.org/10. 1186/s12909-018-1345-5.

29. Terry K. Infectious disease docs in short supply yet 'critical' for COVID-19 crisis. 2020. https://www.medscape.com/viewarticle/ 926387.

Publisher's Note Springer Nature remains neutral with regard to jurisdictional claims in published maps and institutional affiliations. 